\title{
The Early Chemical Evolution of Dwarf Irregular Galaxies
}

\author{
Gerhard Hensler $^{1,2}$, Simone Recchi ${ }^{1,2}$, Joachim Köppen ${ }^{2,3}$, Andreas \\ Rieschick ${ }^{2}$ \\ 1) Institute of Astronomy, University Observatory, \\ Türkernschanzstr. 17, A-1080 Vienna, Austria \\ 2) Institute of Theoretical Physics and Astrophysics, University of Kiel, \\ Olshausenstr. 40, D-24098 Kiel, Germany \\ 3) UMR 7550, Observatoire Astronomique de Strasbourg, \\ 11 rue de l'Universit, F-67000 Strasbourg, France
}

\begin{abstract}
Investigations have been performed to understand the age of dwarf irregular galaxies from the point of view of their presently low chemical abundances and the enhanced star-formation rate. While for a few objects, like e.g. I $\mathrm{Zw} 18$, their assumed relative youth can be verified by models taking a double-starburst behavior into account, those dIrrs with old underlying stellar populations have passed the regime of observed $\mathrm{N} / \mathrm{O}-\mathrm{O}$ values within almost 2 Gyrs after their formation even with gas loss by means of supernova-driven galactic winds. Therefore alternative processes like gas infall must be invoked.
\end{abstract}

\section{Introduction}

Dwarf galaxies (DGs) serve as cornerstones for understanding the evolution of galaxies as well as for determining the basic cosmological model. On the one hand, they are invoked to be the building blocks for the accumulation of galaxy mass in the hierarchical CDM cosmology, while, on the other hand, their evolutionary paths from the formation to their fading and even their requested disappearance differ substantially. They seem to form at all cosmological epochs, by different processes and from different sources. Because of their low binding energy their evolution is strongly affected by internal and external energetic events thus leading to a wide variety of morphological types.

Dwarf elliptical galaxies e.g. are an extremely common and astrophysically interesting class of galaxies, because they seem to have formed preferably in dense environments. Objects of their extension to lower masses, the dwarf spheroidals (dSphs), are at present only detectable within the local group, where they appear as satellite galaxies in the vicinity of the large spiral galaxies (gSs), M31 and the Milky Way. Although their gas is easily evacuated by accumulated supernova explosions so that many dSphs have ceased their star formation (SF) showing only an intermediate-age stellar population (Hodge 1989, Grebel 1997), also more recent SF events have occurred in some objects. Those have experienced two to three short and episodic SF epochs interrupted by longer periods of quiescence. There does not exist a fully homogeneous picture of their evolution, because in some dSphs the metallicity increases with time, indicating that gas was partly kept in the system, while this is not the case in others. 
Equivalently, the gas-rich variant of DGs, dwarf irregulars (dIrrs), show at present a large variety of SF rates from low to extraordinarily high values as e.g. in starbursts (SBDGs). Another mystery of dIrrs are their unusual abundances. As already demonstrated by Pagel (1985) some dIrrs, though with $\mathrm{O}$ abundances below 1/10 solar, show also low N/O ratios of up to 0.7 dex smaller than in $\mathrm{gSs}$, with a large scatter and no significant correlation with $\mathrm{O} / \mathrm{H}$. Their regime of $\mathrm{N} / \mathrm{O}-\mathrm{O}$ values overlap with those of $\mathrm{H}$ II regions in the outermost disk parts of gSs at around $12+\log (\mathrm{O} / \mathrm{H})=8.0 \ldots 8.5$ (van Zee, Salzer, \& Haynes 1998a). These two effects, namely, high SF rates that cannot be maintained for a Hubble time, and low metallicities challenge the picture of dIrrs to be unevolved systems in the early stage after their formation.

More detailed observations during the past years, however, have unveiled old underlying stellar components in most dIrrs like e.g. in NGC 1569 (Heckman et al. 1995) or NGC 1705 (Meurer et al. 1992). The newly formed stellar associations are often very massive and compact, called super star clusters (SSCs). Two possibilities are plausible to reduce the metal abundances in the presence of old stellar populations: loss of metal-enriched gas or infall of metal-poor to even pristine intergalactic gas. Garnett (2002) and van Zee (2001) have shown that the effective yield in dIrrs decreases for low-mass galaxies, what means that their element abundances, in particular $\mathrm{O}$ measured in $\mathrm{H}$ II regions, are smaller than those released by a stellar population and confined in a closed box. Nevertheless, three fundamental questions remain open: 1) Where would a forming dIrr be located in the $\mathrm{N} / \mathrm{O}-\mathrm{O}$ diagram during its early evolution? 2) If dIrrs are old can they still reach the observed N/O-O regime? And 3) Which physical process triggers such enormously high SF rates, while it is expected that due to the low gravitational trough SF should work extremely self-regulated in DGs (Köppen, Theis, \& Hensler 1995, 1998)?

\section{The Early Phases of dIrr Chemical Evolution}

To study the first question we have performed chemo-dynamical numerical models of dIrrs' evolution. The chemo-dynamical prescription properly takes the energetics and dynamics of different gas phases, their mutual interactions and those of the star-gas coupling into account. The chemo-dynamical formulation in 1d and 2d dynamics with the "materialistic" and "energetic" equations is described e.g. in Theis, Burkert, \& Hensler (1992) and Samland, Hensler, \& Theis (1997), respectively. Its effects by application to dIrrs are various and demonstrated e.g. in Hensler, Rieschick, \& Köppen (1999), Rieschick \& Hensler (2000), Hensler \& Rieschick (2002), Rieschick \& Hensler (2003).

Our model starts from $10^{10} \mathrm{M}_{\odot}$ baryonic mass with a Plummer-Kuzmin distribution over a radius of $20 \mathrm{kpc}$ and a Dark Matter halo of $10^{11} \mathrm{M}_{\odot}$ distributed according to Burkert (1995). The angular momentum is set according to the spin parameter $\lambda=0.05$. These initial conditions are chosen to represent a protogalactic gas cloud that has been ionized by the metagalactic radiation field until its cooling dominates and it collapses dissipatively. In addition, only the initial stellar mass function has to be fixed as a free parameter, because the other processes are determined by self regulation and formulated in accordance to results from empirical or theoretical studies. 


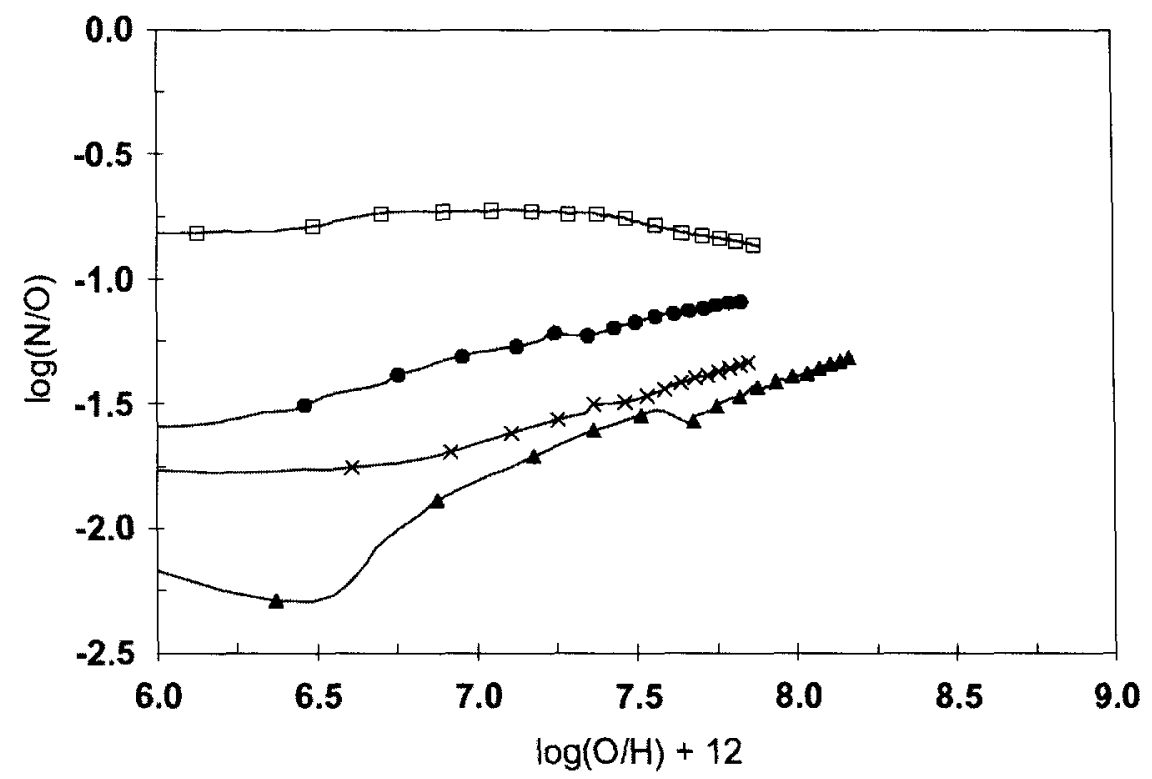

Figure 1. The $\mathrm{N}$ and $\mathrm{O}$ enhancement during the early evolution (2 Gyrs) for different regions of a representative dIrr that assembles a disk of $10^{9} \mathrm{M}_{\odot}$ : galactic disk (filled dots), central $1.5 \mathrm{kpc}$ (open squares), a $5 \mathrm{kpc}$ sphere without the disk (triangles), whole model galaxy (crosses).

Fig. 1 show the abundance evolution in different regions of the model for the first 2 Gyrs. One can discern that the central region reaches already solar values very rapidly, while the disk enhances $\mathrm{O}$ to $12+\log (\mathrm{O} / \mathrm{H}) \approx 7.8$ at $\log (\mathrm{N} / \mathrm{O})$ between -1.5 and -1.0 . The curve smallest in $\mathrm{N} / \mathrm{O}$ demonstrates that the halo is most $\mathrm{O}$ rich reaching $12+\log (\mathrm{O} / \mathrm{H}) \approx 8.2$.

Conclusively, this chemo-dynamical model shows that dIrrs even with expulsion of $\mathrm{O}$ to the halo should evolve rapidly to the right-hand side in the $\mathrm{N} / \mathrm{O}-\mathrm{O}$ diagram only touching the observed regime of dIrrs at their upper N/O values. This evolutionary path is thus almost equal to the evolutionary tracks published for damped Ly $\alpha$ systems by Henry, Edmunds, \& Köppen (2000). For the present conditions of dIrrs this means, that if they contain an old stellar population that has polluted the ISM with the elements under consideration and even with different effective yields due to gas loss by galactic winds, they are expected not to be located in the observed range of N/O-O.

\section{The Evolution of $\mathrm{I} \mathrm{Zw} 18$}

I Z 18 was considered until recently as one of the best candidates for a truly "young" galaxy. However, recent HST observations were deep enough to resolve faint stars and thus to reveal an older stellar population. Quoting Ostlin (2000) "We can thus conclude that all these different studies on independent sets of data in various spectral regions give the same answer: that I $\mathrm{Zw} 18$ is an old 


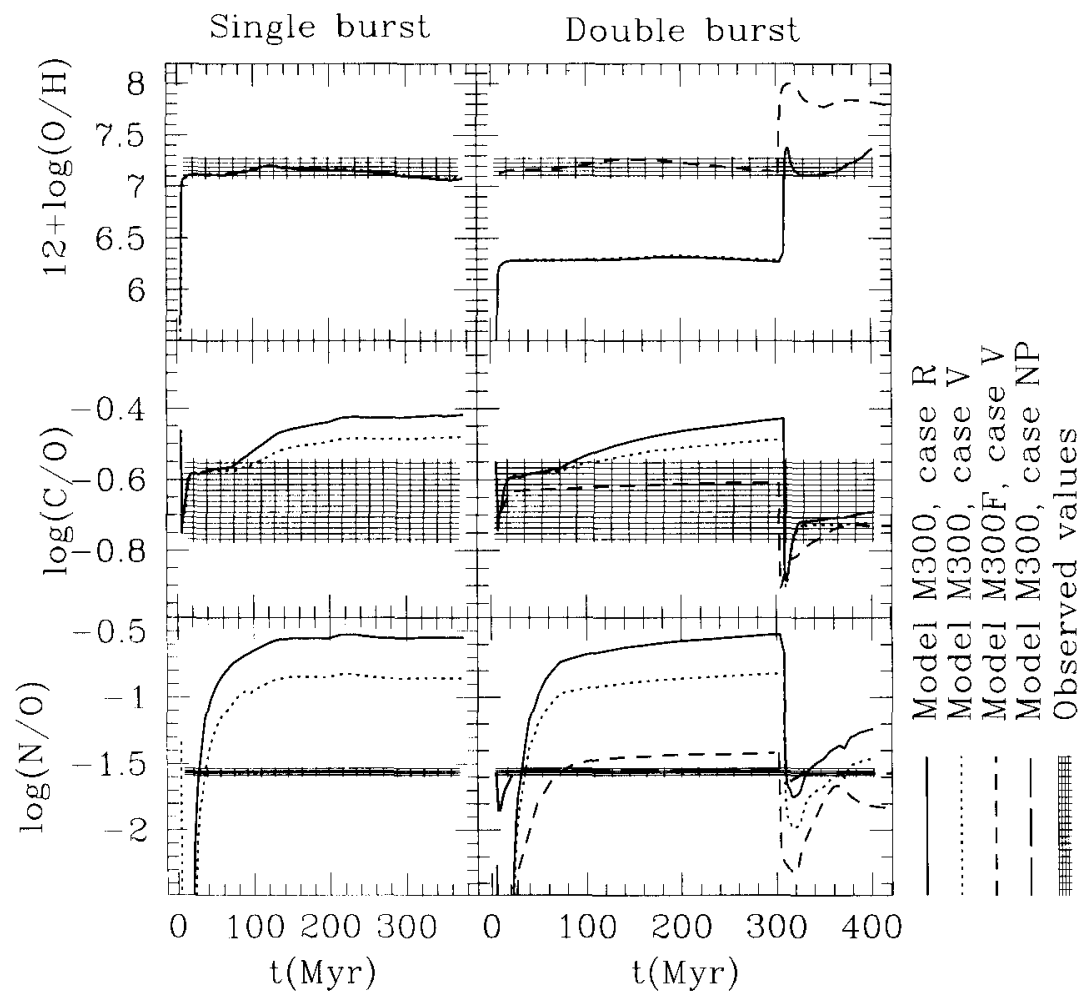

Figure 2. C, N, O evolution for the single-burst model (left panels) and double burst ones (right panels). Both sets of yields from intermediate-mass stars from Renzini \& Voli (1981) (case R) and from van den Hoek \& Groenewegen (1997) (case V) are implemented. Dashed line is a model with a flat $(\mathrm{x}=0.5)$ IMF, whereas all the other models assume a Salpeter IMF. Also shown, in the bottom-right panel, the evolution of $\mathrm{N} / \mathrm{O}$ for a model (model NP) in which we assumed an "ad hoc" production of primary $\mathrm{N}$ in massive stars. The superimposed dashed areas represent the observational values found in literature for IZw18.

galaxy". But, "how old is old?". To address this question, we performed 2-D chemo-dynamical simulations of a model resembling $I Z_{w} 18$, in order to put constraints on the past SF history of this object. Our models consider two possible evolutionary scenarios: bursting vs. continuous SF.

Two instantaneous bursts of SF are separated by a quiescent period of 300 Myr. Owing to the low luminosity of the first burst, a galactic wind does not develop. After $300 \mathrm{Myr}$, most of the gas mass in the central region is cold and dense, thus the onset of a second burst is likely. This second burst creates a strong galactic wind and the metals produced are easily channeled along the galactic chimney. We tested different chemical evolution yields and different IMFs. We conclude that, in order to reproduce the abundances and abundance ratios observed in I Zw 18, the age of the second burst of SF should be not more than 7 Myr (Fig. 2). Details about this set of calculations can be found 
in Recchi et al. (2002). Also remarkable is that most of the metal-enriched gas locked inside the galactic region (i.e. the region in which stars are present) cools in a relatively short time-scale, at variance with previous similar estimates.

We adopt the SF rate as suggested by Aloisi, Tosi \& Greggio (1999), namely constant for almost 300 Myr with $6 \times 10^{-3} \mathrm{M}_{\odot} \mathrm{y}^{-1}$, and superimpose a second, more vigorous burst of $3 \times 10^{-2} \mathrm{M}_{\odot} \mathrm{y}^{-1}$, lasting for $5 \mathrm{Myr}$. The favored age of the last burst is, assuming this SF history, around 15 Myr. As discernible in Fig. 2 the model $\mathrm{N} / \mathrm{O}$ is larger than observed. This is due to the fact that the last burst of SF is only 5 times more intense than the average SFR and lasts for only $5 \mathrm{Myr}$. The $\mathrm{O}$ production is not enough to compensate the $\mathrm{N}$ coming from intermediate-mass stars. There are indications (Chiappini et al. 2003) that the sets of yields of van den Hoek \& Groenewegen (1997) overestimate N. Recent models of stellar evolution with rotation (Meynet \& Maeder 2002) predict less $\mathrm{N}$, although they do not take into consideration the third dredge-up. These models are much more promising in order to reproduce the chemical evolution of metal-poor systems.

We test also the effect of a cloudy medium, in which gas exchange processes by means of condensation and evaporation are acting between the cloud and the surrounding diffuse medium and to how it changes the chemical evolution. The time-scale for a complete evaporation of clouds is around $30 \mathrm{Myr}$. Only clouds outside the central region can survive after the first tens of Myr. In these models thus less energy is available to drive the galactic wind, but the difference with the "smooth" models is still small.

In conclusion, we can state that the last burst of SF in I $\mathrm{Zw}_{\mathrm{w}} 18$ is very young (less than $15 \mathrm{Myr}$ ), in agreement with other estimates (Hunt et al. 2003; Mas-Hesse \& Kunth 1999). Stars older that $300 \mathrm{Myr}$ are not required in order to reproduce the chemical composition of the neutral and ionized medium.

\section{The Rejuvenation of dIrrs by Gas Infall}

Instead of reducing the element abundances by means of galactic winds driven by different episodes of SBs (e.g. in the models by Garnett (1990), Pilyugin (1992), Marconi, Matteucci, \& Tosi (1994)) it is also plausible that infall of gas reduces the abundances. Moreover, the SF triggered by gas infall would fit into this scenario. This seems to be proven observationally, because of the growing evidence that most SBDGs are surrounded by large $\mathrm{H}$ I reservoirs (e.g. NGC 4449: Hunter et al. 1998; I Zw 18: van Zee et al. 1998b). At least, their exist two clear indications that such gas like in the case of He 2-10 collides with the luminous stellar body of the dIrr producing several knots of SSCs (Kobulnicky et al. 1995) or like in NGC 1569 falls in (Stil \& Isreal 2003) where two massive star clusters are ignited. This infall scenario has to be elaborated (Hensler et al. 1999; Hensler et al. 2003).

\section{Acknowledgments}

Parts of this project are supported by the Deutsche Forschungsgemeinschaft (DFG) under grants no. HE 1487/23 (AR) and HE 1487/28 (SR). SR acknowledges financial support from the Alexander von Humboldt Foundation. The 
attendance of the GA2003 in Sydney was enabled for GH by a DFG travel grant no. HE $1487 / 31$. GH also thanks the organizers of JD 15 for their invitation.

\section{References}

Aloisi, A., Tosi, M., \& Greggio, L. 1999, AJ, 118, 302

Burkert, A. 1995, ApJ, 447, L25

Chiappini, C., Romano, D., \& Matteucci, F. 2003, MNRAS, 339, 63

Grebel, E. 1997, Rev. Modern Astron., 10, 29

Garnett, D.R. 1990, ApJ, 360, 142

Garnett, D.R. 2002, ApJ, 581, 1019

Heckman, T.M., Dahlem, M., Lehnert, M.D., et al. 1995, ApJ, 448, 98

Henry, R.B.C., Edmunds, M.G., \& Köppen, J. 2000, ApJ, 541, 660

Hensler, G. \& Rieschick, A. 2002, in ASP Conf. Ser. Vol. 285, Modes of Star Formation, ed. E. Grebel \& W. Brandner, (San Francisco: ASP), 341

Hensler, G., Rieschick A., \& Köppen, J. 1999, in ASP Conf. Ser. Vol. 187, The Evolution of Galaxies on Cosmological Timescales, ed. J. Beckman \& T.J. Mahoney, (San Francisco: ASP), 214

Hensler, G., Köppen, J., Hirche, S., Pflamm, J., \& Rieschick, A., 2003, in Symp. 217, ed. P.-A. Duc et al., 178

Hodge, P.W. 1989, ARA\&A, 27, 139

Hunt, L., Thuan, T.X., \& Izotov, Y.I. 2003, ApJ, 588, 281

Hunter, D.A. et al. 1998, ApJ, 495, 47

Kobulnicky, H.A. et al. 1995, AJ, 110, 116

Köppen, J., Theis, C., \& Hensler, G. 1995, A\&A, 296, 99

Köppen, J., Theis, C., \& Hensler, G. 1998, A\&A, 328, 121

Mas-Hesse, J.M., \& Kunth, D. 1999, A\&A, 349, 765

Marconi, G., Matteucci, F., \& Tosi, M. 1994, MNRAS, 270, 35

Meurer, G.R., Freeman, K.C., Dopita, M.A., et al. 1992, AJ, 103, 60

Meynet, G., \& Maeder, A. 2002, A\&A, 390, 561

Östlin, G. 2000, ApJ, 535, L99

Pagel, B.E.P. 1985, in "Production and Distribution of C,N,O Elements", ed. I.J. Danziger, F. Matteucci, \& K. Kjär, 155

Pilyugin, L.S. 1992, A\&A, 260, 58

Recchi, S., Matteucci, F., D'Ercole, A., \& Tosi, M. 2002, A\&A, 384, 799

Renzini, A. \& Voli, M. 1981, A\&A, 94, 175

Rieschick, A., \& Hensler, G. 2000, in ASP Conf. Ser. Vol. 215, Proc. III. Haro Conf., Cosmic Evolution and Galaxy Formation: Structure, Interactions, and Feedback, ed. J. Franco et al., (San Francisco: ASP), 130

Rieschick, A., \& Hensler, G. 2003, Ap\&SS, 284, 86

Samland, M., Hensler, G., \& Theis, Ch. 1997, ApJ, 476, 544

Stil, J.M. \& Isreal, F.P. 2003, A\&A, 392, 473

Theis, Ch., Burkert, A., \& Hensler, G. 1992, A\&A, 265, 465

van den Hoek, L.B., \& Groenewegen, M.A.T. 1997, A\&AS, 123, 305

van Zee, L. 2001, AJ, 121, 2003

van Zee, L., Salzer, J.J., \& Haynes M.P. 1998a, ApJ, 497, L1

van Zee, L., Westphal, D., Haynes M.P., \& Salzer, J.J. 1998b, AJ, 115, 1000 